\title{
NOUVELLE
}

\section{Un nouvel espoir pour traiter les infections persistantes à Mycobacterium abscessus ?}

Clément Raynaud, Laurent Kremer
CNRS UMR 9004, Institut de Recherche en Infectiologie de Montpellier (IRIM), Université de Montpellier, 1919 route de Mende, 34293 Montpellier, France

laurent.kremer@irim.cnrs.fr
> Les bactéries du genre Mycobacterium, ou mycobactéries, se divisent en deux groupes principaux: les mycobactéries à croissance lente, dont font partie plusieurs espèces pathogènes strictes telles que $M$. tuberculosis ou $M$. leprae, respectivement responsables de la tuberculose et de la lèpre, et les mycobactéries à croissance rapide, qui comprennent des espèces pathogènes opportunistes telles que $M$. fortuitum ou M. abscessus [1]. Cette dernière est responsable d'infections cutanées ou des tissus mous, mais les principales formes sévères sont pulmonaires, notamment chez des personnes ayant des prédispositions particulières et des maladies sous-jacentes (bronchiectasie, mucoviscidose, etc.). Chez les patients atteints de mucoviscidose, les infections pulmonaires causées par des mycobactéries non tuberculeuses sont relativement fréquentes (10 à $14 \%$ des cas), et plus de $50 \%$ d'entre elles sont causées par le complexe M. abscessus [2]. Ce complexe comprend trois sousespèces, M. abscessus stricto sensu, $M$. bolletii et $M$. massiliense, qui diffèrent par leur sensibilité à certains antibiotiques. Toutes trois peuvent exister sous la forme de deux morphotypes distincts : 1) le morphotype lisse, qui se caractérise par l'abondante production de lipides de surface, les glycopeptidolipides (GPL), lesquels participent à la motilité des bacilles sur un milieu gélosé et à la formation de pellicules à l'interface liquide/air, apparentées à des biofilms, et 2) le morphotype rugueux, qui se caractérise par la faible production ou l'absence de GPL à la surface des bactéries. Le caractère très hydrophobe des formes rugueuses stimule la formation d'agrégats bactériens aboutissant à la formation de cordes, très difficiles à dissocier et résistantes à la phagocytose par les macrophages et les granulocytes neutrophiles [3] $(\rightarrow)$ ainsi qu'à de nombreux agents antiseptiques.

\section{$(\rightarrow)$ Voir la Nouvelle de A. Bernut et al., $m / s n^{\circ} 5$, mai 2014, page 499} Par ailleurs, l'infection par la forme rugueuse de ces bactéries est souvent associée à un déclin de la fonction pulmonaire chez les patients atteints de mucoviscidose [4], et cette forme est considérée comme la plus virulente dans divers modèles animaux.

\section{Une espèce hautement résistante} à de nombreux antibiotiques

M. abscessus est naturellement résistante à la plupart des familles d'antibiotiques utilisées en clinique, y compris à la quasi-totalité des agents antituberculeux, ce qui complique considérablement le traitement des infections par cette bactérie. Si la thérapie comprend généralement l'administration d'une 
$\beta$-lactamine (imipénème ou céfoxitine) associée à un macrolide (clarithromycine ou azithromycine) et à un aminoglycoside (amikacine) pendant plusieurs mois, le risque de rechute reste très élevé du fait d'un terrain propice à l'infection pulmonaire ou de l'induction de mécanismes de résistance aux antibiotiques.

La résistance de M. abscessus aux antibiotiques implique de nombreux mécanismes de résistance innée [1]: 1) une panoplie d'enzymes qui modifient l'antibiotique (acétyltransférases, $\beta$-lactamase et ADPribosyl-transférase, qui inactivent les aminoglycosides, $\beta$-lactamines et rifamycines, respectivement), 2) des enzymes qui modifient la cible des antibiotiques (méthyl-transférase qui modifie la sous-unité $23 \mathrm{~S}$ du ribosome, empêchant la fixation des macrolides), et 3 ) des pompes à efflux capables de transporter certains antibiotiques, comme la bédaquiline et la clofazimine, vers l'extérieur de la bactérie. A cela s'ajoutent des mutations acquises durant les périodes prolongées de traitement des patients infectés. En pratique clinique, des mutations ont notamment été observées dans les gènes rrs et rrl, codant les ARNr 165 et 235 , et impliqués respectivement dans la résistance aux aminoglycosides et aux macrolides. L'ensemble de ces mécanismes de résistance englobe toutes les classes d'antibiotiques utilisées pour traiter les infections pulmonaires à M. abscessus. Dès lors, il apparaît urgent de développer de nouveaux traitements, en recourant à des molécules existantes déjà utilisées contre d'autres maladies (stratégie de drug repositioning) ou en identifiant de nouvelles entités chimiques agissant sur de nouvelles cibles thérapeutiques.

\section{Le transporteur d'acides mycoliques}

MmpL3 : une nouvelle cible d'intérêt pharmacologique

La paroi des mycobactéries regorge de lipides complexes participant à la virulence. Ils sont généralement synthétisés dans le cytoplasme et exportés à la surface du bacille par l'intermédiaire de transporteurs membranaires de la famille MmpL (mycobacterial membrane protein large) [5]. Les GPL, par exemple, sont transportés à travers la membrane plasmique par MmpL4a et MmpL4b, tandis que d'autres MmpL participent à l'efflux de certains antibiotiques. Les acides mycoliques sont des acides gras essentiels à très longue chaîne carbonée, qui sont synthétisés dans le cytoplasme sous la forme de tréhalose mono-mycolate (TMM). Le TMM est transporté par la protéine MmpL3 vers la mycomembrane, où il servira de substrat aux mycolyl-transférases du complexe Ag85 (antigen 85), soit pour être estérifié sur l'arabinane de l'arabinogalactane, soit pour servir à la synthèse de tréhalose di-mycolate (TDM), qui s'insèrera dans le feuillet externe de la mycomembrane (Figure 1). Du fait de la nature essentielle du gène mmpL3 pour la survie des mycobactéries et de l'absence de gènes orthologues chez les mammifères, de nombreuses études, réalisées tout d'abord chez M. tuberculosis, ont permis d'identifier plusieurs familles de composés capables

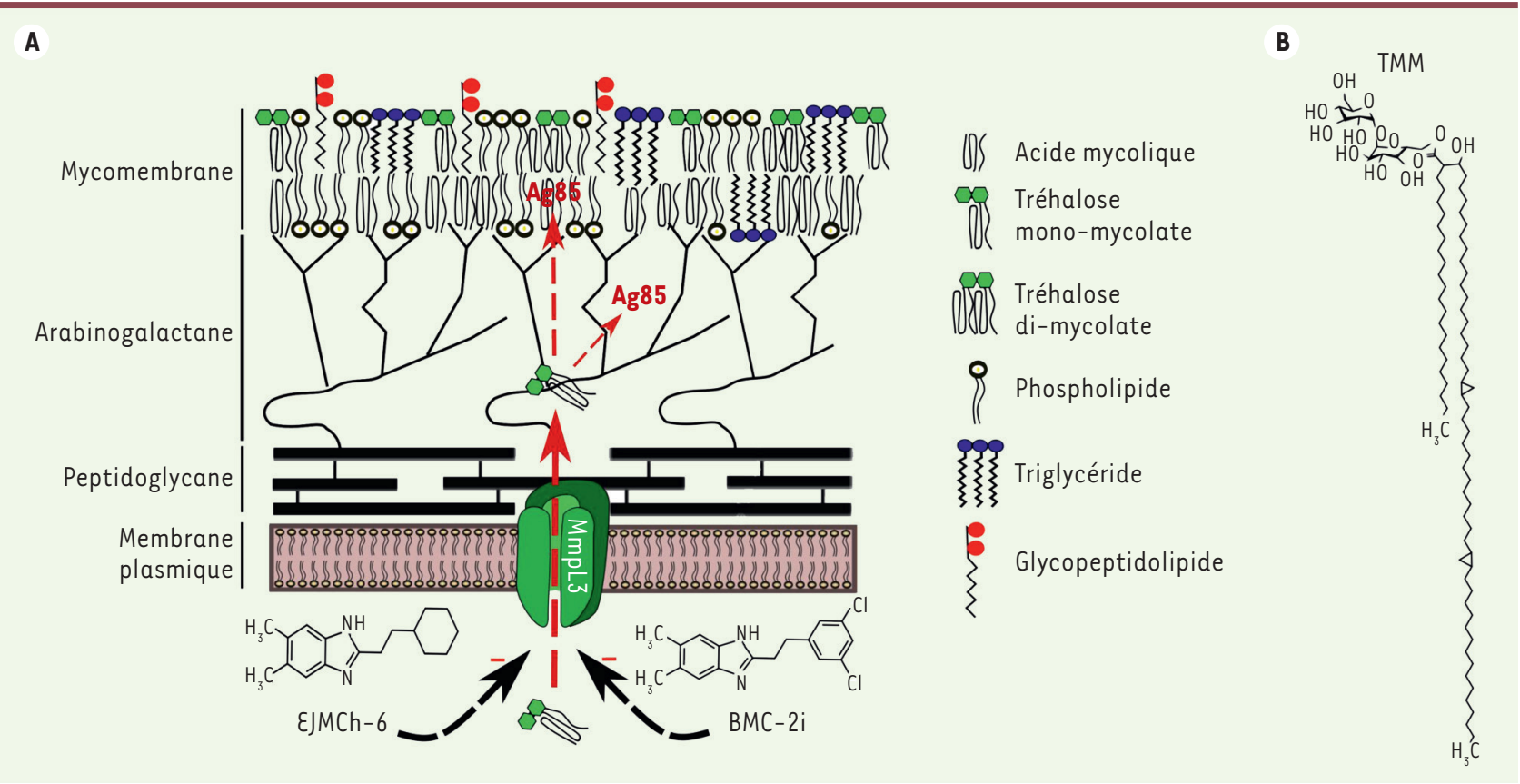

Figure 1. Vue schématique de la paroi de Mycobacterium abscessus et mode d'action des inhibiteurs de MmpL3. (A) EJMCh-6 et BMC-2i inhibent le transport du tréhalose mono-mycolate (TMM), qui sert de substrat aux protéines du complexe Ag85 pour mycolyler l'arabinogalactane ou produire du tréhalose di-mycolate (TDM), qui s'insère dans la membrane bactérienne externe. (B) Structure du TMM. 


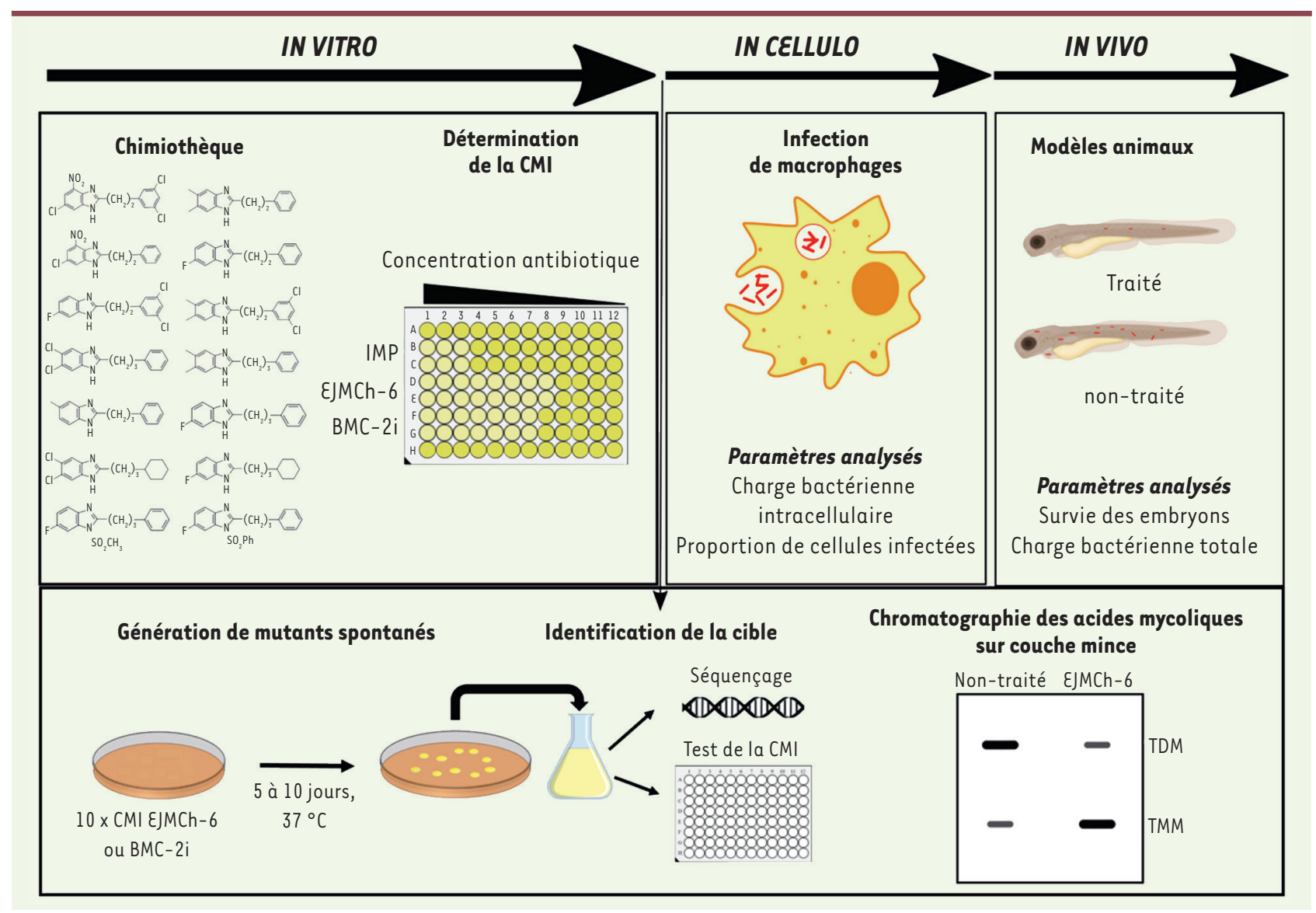

Figure 2. Principales étapes depuis l'identification des composés phares $\varepsilon$ JMCh-6 et BMC-2i à partir d'une « chimiothèque 》 de dérivés du benzimidazole jusqu'aux tests précliniques chez l'embryon du poisson-zèbre (Danio rerio). L'approche utilisée pour l'identification de la cible de ces composés (le transporteur d'acides mycoliques MmpL3), combinant à la fois des études génétiques et biochimiques, est également représentée. IMP, imipénème ; CMI, concentration minimale inhibitrice.

d'inhiber la translocation du TMM en bloquant l'activité flippase ${ }^{1}$ de $M m p L 3$. Récemment, plusieurs entités chimiques présentant une forte activité contre $M$. abscessus et inhibant MmpL3 ont été décrites, incluant des dérivés de pipéridinol [6], des indole-2-carboxamides [7] et des dérivés du benzimidazole [8].

\section{Activité des benzimidazoles}

sur les formes intracellulaires

et extracellulaires de M. abscessus

Les benzimidazoles ont d'abord été synthétisés dans le but d'inhiber la croissance de M. tuberculosis [9], ce qui suggérait la possibilité d'un effet comparable

1 Les flippases sont des enzymes qui participent au maintien de l'asymétrie de la double couche lipidique de la membrane cytoplasmique en transportant des lipides d'une couche à l'autre. chez M. abscessus. Le criblage d'une chimiothèque de 34 dérivés du benzimidazole a permis d'identifier deux composés phares, EJMCh- 6 et BMC-2i, possédant des concentrations minimales inhibitrices très basses $(0,125$ et $0,25 \mu \mathrm{g} / \mathrm{mL}$, respectivement) vis-à-vis de la souche de référence de M. abscessus, CIP104536 (Figure 2). हJMCh- 6 montre également une très bonne activité contre un large panel d'isolats cliniques de cette bactérie, d'origines diverses, notamment issus de patients atteints de mucoviscidose. Aucune différence de susceptibilité à EJMCh-6 n'a été observée entre les morphotypes lisse et rugueux de M. abscessus. Par ailleurs, l'exposition à EJMCh-6 de macrophages humains infectés par cette bactérie se traduit par une très nette diminution $\mathrm{du}$ nombre de bactéries intracellulaires à des concentrations de ce composé pour lesquelles aucune cytotoxicité apparente n'est observée sur des macrophages sains. Enfin, l'efficacité thérapeutique du composé $\varepsilon J M C h-6$ a été testée avec succès chez l'embryon du poisson-zèbre (Danio rerio) infecté par le variant rugueux de M. abscessus, qui est la forme la plus virulente de la bactérie dans ce modèle animal [3]. Le traitement s'accompagne d'une amélioration considérable de la survie des embryons infectés, consécutive à une nette diminution de la charge bactérienne dans les embryons (Figure 2). Pour confirmer que MmpL3 est la cible de $\varepsilon J M C h-6$ et de BMC-2i, des mutants bactériens spontanés résistants à ces deux composés ont été sélectionnés in vitro, et le séquençage de $\mathrm{mmp} L 3$ a révélé l'existence de neuf mutations ponctuelles 
distinctes chez ces mutants (Figure 2). De plus, l'introduction de différents allèles de $m m p L 3$ renfermant ces mutations ponctuelles dans une souche sensible à $\varepsilon M M C h-6$ confère à cette souche une résistance accrue à $\varepsilon J M C h-6$ et $B M C-2 i$ [8]. Par ailleurs, l'analyse du profil lipidique d'une culture de M. tuberculosis traitée avec हJMCh-6 s'accompagne d'une diminution de la synthèse de TDM au profit d'une accumulation du TMM [9]. Ainsi, l'ensemble de ces résultats indique que हJMCh-6 inhibe la translocation du TMM en ciblant MmpL3 chez les mycobactéries.

Vers de nouveaux développements précliniques?

Plusieurs classes d'inhibiteurs de MmpL3 ont montré une activité synergique avec des molécules utilisées en clinique, en particulier les $\beta$-lactamines, dans des tests in vitro [10], ce qui permettrait d'utiliser in vivo des doses d'antibiotique réduites et d'obtenir une activité antimycobactérienne similaire, voire meilleure que lorsque ces molécules sont utilisées individuellement. Une conséquence importante de la réduction des doses d'antibiotiques efficaces est de limiter à la fois les effets secondaires liés à une exposition prolongée à ces antibiotiques, mais aussi de prévenir l'émergence de souches résistantes. Cependant, l'activité synergique de EJMCh-6 et de BMC-2i avec d'autres antibiotiques (imipénème, céfoxitine) utilisés en clinique reste à vérifier expérimentalement.
Enfin, grâce à la structure cristallographique de la protéine MmpL3 de M. smegmatis publiée récemment [11], et à un modèle prédictif de celle de $M$. abscessus [8], il devient possible d'entreprendre des études de type relation structureactivité afin de produire, en lien avec des chimistes, de nouveaux dérivés de EJMCh-6 aux propriétés pharmacologiques et physicochimiques améliorées. Une prochaine étape dans l'évaluation de ces composés consistera à évaluer leurs propriétés pharmacocinétiques et toxicologiques dans un modèle animal plus complexe que le poisson-zèbre. Depuis quelques années en effet, plusieurs modèles murins immunodéprimés ont été décrits pour étudier la physiopathologie infectieuse de M. abscessus. Récemment, un modèle de souris immunocompétentes, $\mathrm{C} 3 \mathrm{HeB} / \mathrm{Fe}$ ), propice à une infection par M. abscessus persistant jusqu'à 25 jours, a été rapporté et utilisé avec succès pour démontrer l'activité thérapeutique de la bédaquiline in vivo [12]. Les résultats très prometteurs de tous ces travaux ouvrent désormais la voie d'une alternative thérapeutique contre les infections pulmonaires à $M$. abscessus. $\diamond$

New hopes for the treatment

of persistent Mycobacterium abscessus infections?

\section{REMERCIEMENTS}

Ce travail a bénéficié d'une aide des associations Vaincre la mucoviscidose et Grégory Lemarchal ainsi que du programme Équipe FRM de la Fondation pour la recherche médicale.

\section{LIENS D'INTÉRÊT}

Les auteurs déclarent n'avoir aucun lien d'intérêt concernant les données publiées dans cet article.

\section{RÉFÉRENCES}

1. Johansen MD, Herrmann JL, Kremer L. Non-tuberculous mycobacteria and the rise of Mycobacterium abscessus. Nat Rev Microbiol 2020 ; https://doi. org/10.1038/s41579-020-0331-1.

2. Mougari F, Guglielmetti L, Raskine L, et al. Infections caused by Mycobacterium abscessus: epidemiology, diagnostic tools and treatment. Exp Rev Anti-Inf Ther 2016 ; 14 : 1139-54.

3. Bernut A, Herrmann JL, Lutfalla G, Kremer L. Les cordes mycobactériennes: un nouveau moyen d'échappement au système immunitaire? Med Sci (Paris) $2014 ; 30: 499-502$.

4. Catherinot $\varepsilon$, Roux AL, Macheras $\varepsilon$, et al. Acute respiratory failure involving an $R$ variant of Mycobacterium abscessus.J Clin Microbiol 2009 ; 47 : 271-4.

5. Chalut C. MmpL transporter-mediated export of cell-wall associated lipids and siderophores in mycobacteria. Tuberculosis (Edinb) 2016; $100: 32-45$.

6. Dupont C, Viljoen A, Dubar F, et al. A new piperidinol derivative targeting mycolic acid transport in Mycobacterium abscessus. Mol Microbiol 2016 ; 101 : 515-29.

7. Kozikowski A, Onajole OK, Stec J, et al. Targeting mycolic acid transport by indole-2-carboxamides for the treatment of Mycobacterium abscessus infections. J Med Chem 2017 ; 60 : 5876-88.

8. Raynaud C, Daher W, Johansen MD, et al. Active benzimidazole derivatives targeting the $\mathrm{MmpL3}$ transporter in Mycobacterium abscessus. ACS Inf Dis $2020 ; 6: 324-37$.

9. Korycka-Machała M, Viljoen A, Pawełczyk J, et al. ${ }^{1} \mathrm{H}$-Benzo [d] imidazole derivatives affect MmpL3 in Mycobacterium tuberculosis. Antimicrob Agents Chemother 2019; 63 : e00441-19.

10. Li W, Sanchez-Hidalgo A, Jones V, et al. Synergistic interactions of $\mathrm{MmpL} 3$ inhibitors with antitubercular compounds in vitro. Antimicrob Agents Chemother 2017 ; 61 : e02399-16.

11. Zhang B, Li J, Yang X, et al. Crystal structures of membrane transporter $\mathrm{MmpL} 3$, an anti-TB drug target. Cell 2019; 176 : 636-48.el3.

12. Le Moigne V, Raynaud C, Moreau F, et al. Efficacy of bedaquiline, alone or in combination with imipenem, against Mycobacterium abscessus in $\mathrm{C} 3 \mathrm{HeB} / \mathrm{FeJ}$ mice. Antimicrob Agents Chemother 2020 ; 64 : e00114-20.

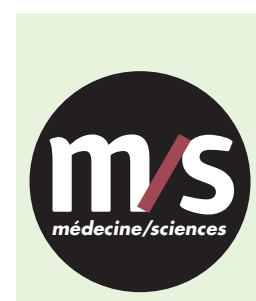

\section{Tarifs d'abonnement $\mathrm{m} / \mathrm{s}-2020$ \\ Abonnez-vous \\ à médecine/sciences}

$>$ Grâce à $m / s$, vivez en direct les progrès des sciences biologiques et médicales

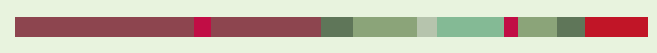

Bulletin d'abonnement page 822 dans ce numéro de $\mathrm{m} / \mathrm{s}$

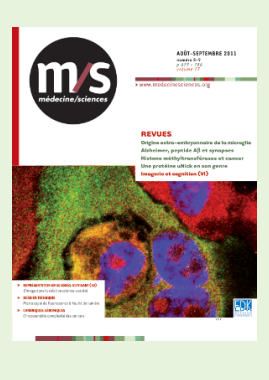

